\title{
ON THE CONTINUITY OF CERTAIN INTERTWINING OPERATORS, CENTRALIZERS, AND POSITIVE LINEAR FUNCTIONALS
}

\author{
MARC A. RIEFFEL
}

Let $A$ be a Banach algebra with a bounded approximate identity, and let $V$ be a Banach $A$-module, that is [8], a Banach space which is an $A$-module in the algebraic sense, and for which $\|a v\| \leqq\|a\|\|v\|$ for all $a \in A, v \in V$. In [8] an important role is played by $\operatorname{Hom}_{A}(A, V)$, the collection of all continuous linear transformations, $T$, from $A$ to $V$ which satisfy

$$
T(a b)=a(T(b)), \quad a, b \in A .
$$

We were thus interested to notice that

THEOREM 1. Every linear transformation $T$ from $A$ to $V$ which satisfies $\left({ }^{*}\right)$ is continuous.

This theorem is an immediate consequence of a lemma found independently by Varopoulos [9] and Johnson [5] which generalizes Cohen's factorization theorem [1]. This lemma was used by Varopoulos to show that if $A$ has a continuous involution, then every positive linear functional on $A$ is continuous. Johnson used the lemma to show that every centralizer on $A$ is continuous, and our Theorem 1 is just a slight generalization of Johnson's result.

Our small contribution to this circle of ideas is to show, by means of a simple device, that the lemma of Varopoulos and Johnson is a corollary of a generalization of Cohen's theorem found by Hewitt [3], Curtis and Figa-Talamanca [2], and Gulick, Liu and van Rooij [4]. Furthermore, a method for simplifying the proof of Cohen's theorem has been found by Koosis [6], and, as he remarks, this method can be used equally well to simplify the proof of the theorem of Hewitt et al. In this way we obtain a quite short proof of the lemma of Varopoulos and Johnson, and so of the three continuity results mentioned above.

We now state the theorem of Hewitt et al. for the case in which $V$ is a left $A$-module. The statement for right modules is entirely analogous.

Theorem 2 (Hewitt et AL.). Let $A$ be a Banach algebra and let $V$

Received by the editors August 14, 1967 and, in revised form, January 19, 1968. 
be a left Banach $A$-module. Suppose that there is a constant, $M$, such that for every $a \in A, v \in V$ and $\epsilon>0$ there exists an element $e \in A$ such that $\|e\| \leqq M,\|a-a e\|<\epsilon$, and $\|v-e v\|<\epsilon$. Then for every $v \in V$ there exist elements $a \in A$ and $w \in V$ such that $v=a w$.

We refer the reader to the paper of Koosis for a proof.

Lemma 1 (Varopoulos-Johnson). Let $A$ be a Banach algebra and $V$ an $A$-module. Suppose that there is a constant, $M$, such that for every $a \in A$, every finite collection $v_{1}, \cdots, v_{k}$ of elements of $V$, and every $\epsilon>0$, there exists an element $e \in A$ such that $\|e\| \leqq M,\|a-a e\|<\epsilon$, and $\left\|v_{j}-e v_{j}\right\|<\epsilon$ for $1 \leqq j \leqq k$. Then for every sequence $\left\{v_{n}\right\}$ of elements of $V$ which converges to 0 there exists $a \in A$ and a sequence $\left\{w_{n}\right\}$ of elements of $V$ which converges to 0 such that $v_{n}=a w_{n}$ for all $n$.

Proof. Let $c_{0}(V)$ be the Banach space of all sequences of elements of $V$ which converge to 0 , with the supremum norm. Then $c_{0}(V)$ is an $A$-module with respect to the evident coordinate-wise action, and it is easily seen that the hypotheses of Lemma 1 ensure that $c_{0}(V)$ satisfies the hypotheses of Theorem 2. Applying this theorem to the element $\left\{v_{n}\right\}$ of $c_{0}(V)$ we obtain the desired elements $a$ and $\left\{w_{n}\right\}$.

Proof of Theorem 1. Let $T$ be a linear transformation from $A$ to $V$ satisfying $\left({ }^{*}\right)$, and let $\left\{a_{n}\right\}$ be a sequence in $A$ converging to 0 . Viewing $A$ as a right module over itself, we can apply the right-hand version of Lemma 1 to obtain $c \in A$ and a sequence $\left\{b_{n}\right\}$ in $A$ with $b_{n}$ converging to 0 , such that $a_{n}=b_{n} c$ for all $n$. Then

$$
T\left(a_{n}\right)=T\left(b_{n} c\right)=b_{n} T(c) \rightarrow 0 .
$$

We remark that Johnson's theorem is just the special case of Theorem 1 in which $V=A$.

For completeness we include Varopoulos' result.

TheOREM 3 (VAROPOULOS). Let $A$ be a Banach algebra with bounded approximate identity and continuous involution. Then every positive linear functional on $A$ is continuous.

Proof. Let $f$ be a positive linear functional on $A$, and let $\left\{a_{n}\right\}$ be a sequence in $A$ converging to 0 . Applying Lemma 1 twice, we obtain $b, c \in A$ and a sequence $\left\{d_{n}\right\}$ in $A$ converging to 0 such that $a_{n}=b d_{n} c$. Thus it suffices to show that the linear functional $a \rightarrow f(b a c)$ is continuous. But by polarization this functional is a linear combination of four functionals of the form $a \rightarrow f\left(e a e^{*}\right)$ for $e \in A$. But such functionals are known to be continuous [7, Theorem 4.5.2]. 


\section{REFERENCES}

1. P. J. Cohen, Factorization in group algebras, Duke Math. J. 26 (1959), 199-206.

2. P. C. Curtis, Jr. and A. Figa-Talamanca, "Factorization theorems for Banach algebras," in Function algebras, edited by Frank T. Birtel, Scott, Foresman and Co., Chicago, Ill., 1966.

3. E. Hewitt, The ranges of certain convolution operators, Math. Scand. 15 (1964), 147-155.

4. S. L. Gulick, T. S. Liu and A. C. M. van Rooij, Group algebra modules. II, Canad. J. Math. 19 (1967), 151-173.

5. B. E. Johnson, Continuity of centralizers on Banach algebras, J. London Math. Soc. 41 (1966), 639-640.

6. P. Koosis, Sur un theorème de Paul Cohen, C. R. Acad. Sci. Paris 259 (1964), 1380-1382. 1960.

7. C. E. Rickart, General theory of Banach algebras, Van Nostrand, New York,

8. M. Rieffel, Induced Banach representations of Banach algebras and locally compact groups, J. Functional Anal. 1 (1967), 443-491.

9. N. T. Varopoulos, Sur les formes positives d'une algèbre de Banach, C. R. Acad. Sci. Paris 258 (1964), 2465-2467.

University of California, Berkeley 